\title{
Statistical Analysis of Instantaneous Frequency Scaling Factor as Derived From Optical Disdrometer Measurements At K/Q Bands
}

\author{
Michael Zemba ${ }^{1}$, James Nessel ${ }^{1}$, Jacquelynne Houts ${ }^{1}$, Lorenzo Luini ${ }^{2}$, Carlo Riva ${ }^{2}$ \\ ${ }^{1}$ NASA Glenn Research Center: Advanced High Frequency Branch, Cleveland, OH, USA \\ ${ }^{2}$ Politecnico di Milano: Dipartimento di Elettronica, Informazione e Bioingegneria, Milan, Italy
}

\begin{abstract}
The rain rate data and statistics of a location are often used in conjunction with models to predict rain attenuation. However, the true attenuation is a function not only of rain rate, but also of the drop size distribution (DSD). Generally, models utilize an average drop size distribution (Laws and Parsons or Marshall and Palmer [1]). However, individual rain events may deviate from these models significantly if their DSD is not well approximated by the average. Therefore, characterizing the relationship between the DSD and attenuation is valuable in improving modeled predictions of rain attenuation statistics. The DSD may also be used to derive the instantaneous frequency scaling factor and thus validate frequency scaling models.
\end{abstract}

Since June of 2014, NASA Glenn Research Center (GRC) and the Politecnico di Milano (POLIMI) have jointly conducted a propagation study in Milan, Italy utilizing the 20 and $40 \mathrm{GHz}$ beacon signals of the Alphasat TDP\#5 Aldo Paraboni payload. The Ka- and Q-band beacon receivers provide a direct measurement of the signal attenuation while concurrent weather instrumentation provides measurements of the atmospheric conditions at the receiver. Among these instruments is a Thies Clima Laser Precipitation Monitor (optical disdrometer) which yields droplet size distributions (DSD); this DSD information can be used to derive a scaling factor that scales the measured $20 \mathrm{GHz}$ data to expected $40 \mathrm{GHz}$ attenuation. Given the capability to both predict and directly observe $40 \mathrm{GHz}$ attenuation, this site is uniquely situated to assess and characterize such predictions.

Previous work using this data has examined the relationship between the measured drop-size distribution and the measured attenuation of the link [2]. The focus of this paper now turns to a deeper analysis of the scaling factor, including the prediction error as a function of attenuation level, correlation between the scaling factor and the rain rate, and the temporal variability of the drop size distribution both within a given rain event and across different varieties of rain events.

Index Terms-drop size distribution, frequency scaling, propagation losses, radiowave propagation.

\section{INTRODUCTION}

Since June of 2014, NASA Glenn Research Center (GRC) and the Politecnico di Milano (POLIMI) have jointly conducted a propagation campaign measuring rain attenuation, scintillation, and other atmospheric propagation phenomena in Milan, Italy using beacon receivers that observe the 20 and 40 GHz beacons onboard the Alphasat TDP\#5 Aldo Paraboni Q/Vband Payload. The receivers are installed at the POLIMI campus in Milan, Italy on the roof of the Dipartimento di Elettronica, Informazione e Bioingegneria (DEIB) building. The Ka- and Q-
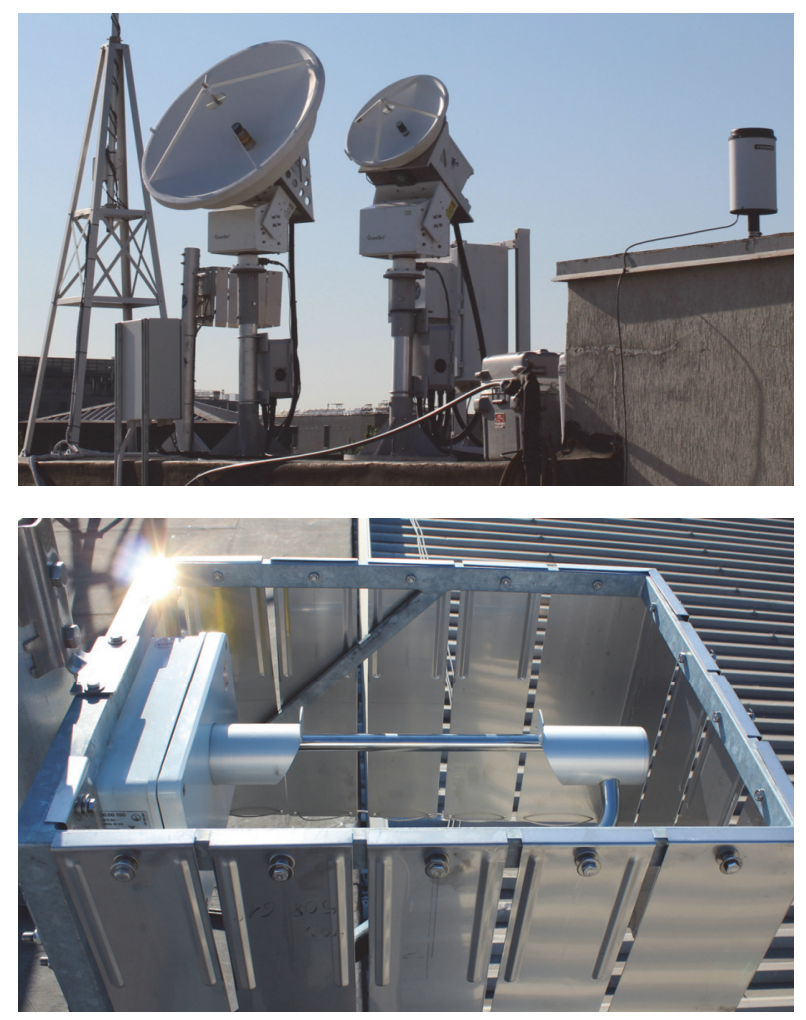

Fig. 1. Photographs of the Ka/Q-band beacon receivers (above) and Thies Clima optical disdrometer (below) at the POLIMI campus in Milan, Italy.

band beacon receivers provide a direct measurement of the link attenuation while collocated meteorological instrumentation provides measurements of the ground level atmospheric conditions. Instrumentation includes the Ka-band and Q-band beacon receivers (Fig. 1, top), a weather station providing measurements of temperature, pressure, humidity, wind speed and wind direction, a tipping bucket, and a laser disdrometer (Fig. 1, bottom). The disdrometer, a Thies Clima Laser Precipitation Monitor, yields droplet size and velocity distributions (DSD, DVD). In particular, the DSD information can be used to calculate specific attenuation at each frequency and thereby derive a scaling factor that translates the measured $20 \mathrm{GHz}$ attenuation data to the expected $40 \mathrm{GHz}$ attenuation. 


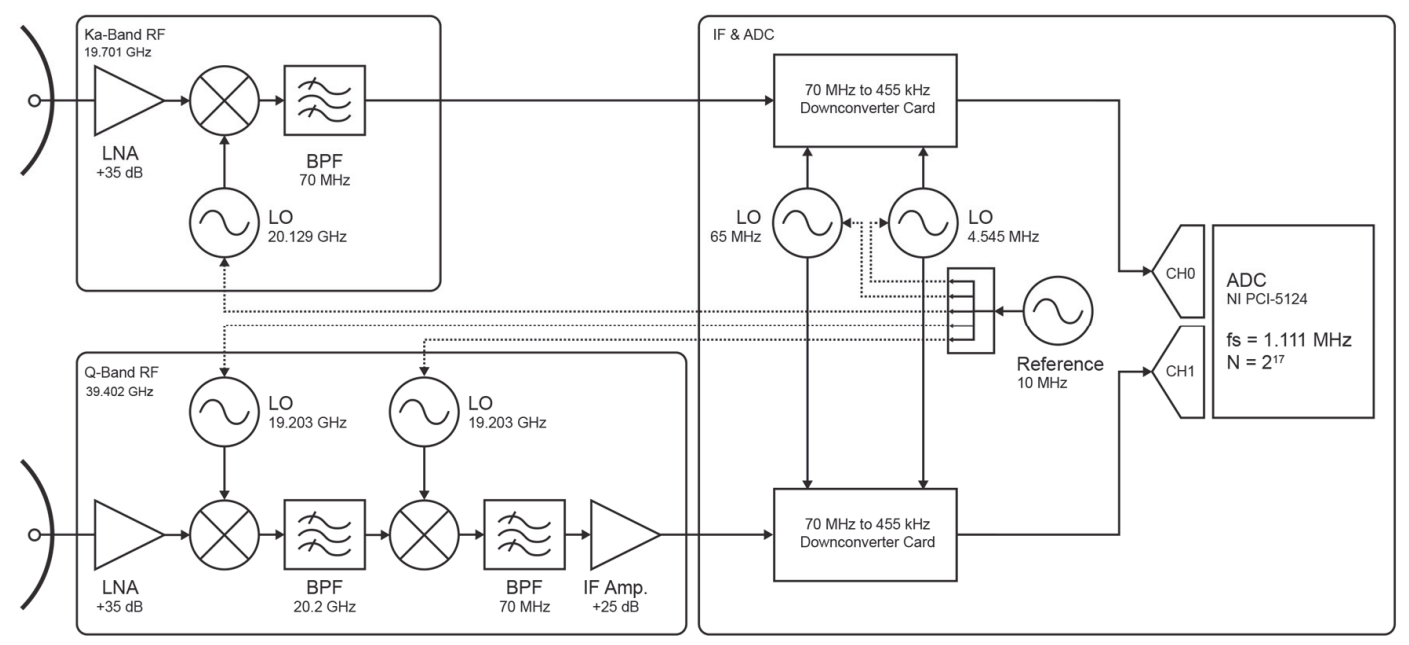

Fig. 2. Block diagram of the beacon receiver design.

Given the capability of the experiment to both predict the scaled $40 \mathrm{GHz}$ attenuation from the $20 \mathrm{GHz}$ data as well as measure the $40 \mathrm{GHz}$ attenuation directly from the beacon receiver, the data may therefore be used to characterize the instantaneous frequency scaling factor in terms of accuracy and statistical variation.

Previous work along these lines has investigated the fundamental relationship between the drop-size distribution and the measured attenuation of the link and some statistical analysis including the probability distribution and seasonal variation of the scaling factor [2]. This paper will further this statistical analysis of the scaling factor by considering the prediction error as a function of attenuation level, correlation between the scaling factor and the rain rate, and the temporal variability of the drop size distribution both within a given rain event and across different types of rain events.

\section{EXPERIMENT DESIGN}

\section{A. Alphasat Ka/Q-Band Beacon Receivers}

The Ka and Q-band beacon receivers, developed at NASA GRC, utilize a $1.2 \mathrm{~m}$ Ka-band and a $0.6 \mathrm{~m}$ Q-band Cassegrain reflector, both with beamwidths of $0.9^{\circ}$. The narrow beamwidth and inclined orbit of Alphasat necessitate active mechanical tracking of the beacons which is accomplished using QuickSet pan-tilt electronic positioners that update the antenna pointing once per minute. Both the $\mathrm{Ka}$ and Q-band signals are downconverted to $70 \mathrm{MHz}$ directly at each antenna's feed inside thermally controlled enclosures. The LNAs, oscillators, mixers, and filters are all thermally stable to within $\pm 0.1^{\circ} \mathrm{C}$. Both signals are then fed into a common IF enclosure (controlled to within $\pm 1.0^{\circ} \mathrm{C}$ ) and further downconverted to $455 \mathrm{kHz}$, as depicted in the block diagram of Fig. 2. All downconversion stages are referenced to a common ultra-stable $10 \mathrm{MHz}$ citrine oscillator. The IF signal is then digitized and processed utilizing a novel frequency estimation routine to record the frequency and power

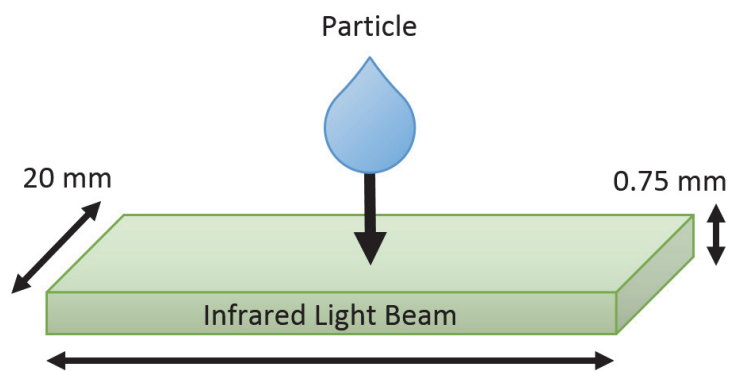

$228 \mathrm{~mm}$

Fig. 3. Diagram depicting laser disdrometer measurement of a precipitating particle [5].

level of the signals [3], and data is recorded at a measurement rate of $8 \mathrm{~Hz}$, as well as averaged once per minute into $1 \mathrm{~Hz}$ measurements. Ultimately, the receivers are able to achieve a dynamic range of approximately $40 \mathrm{~dB}$. The design and characterization of the receivers is further detailed in [4].

\section{B. Thies Clima Disdrometer}

The Thies Clima Laser Precipitation Monitor or disdrometer, pictured in Fig. 1 (bottom), is located alongside the beacon receivers on the DEIB rooftop. It operates by means of an infrared laser diode which generates a $785 \mathrm{~nm}$ beam over an area of $4560 \mathrm{~mm}^{2}$, as depicted in Fig. 3 [5]. A photodiode receiver monitors fluctuations in the received signal power as precipitating particles cross the beam. Using this information, the particle diameter and velocity are derived from the magnitude and duration of the signal attenuation. The observed particles are classified into spectra of 22 diameter bins from $0.125 \mathrm{~mm}$ to $8 \mathrm{~mm}$ and 20 velocity bins from 0 to $10 \mathrm{~m} / \mathrm{s}$ with non-uniform bin widths [5]. Through on-board processing, the instrument also classifies the type of precipitation and intensity. Measurements are recorded in one minute intervals. 


\section{DATA ANALYSIS}

\section{A. Derivation of Scaling Factor}

The scaling factor, and thus the scaled $40 \mathrm{GHz}$ attenuation predictions, are derived from the disdrometer data by using the DSD to calculate the specific attenuation $(\gamma)$ at both $20 \mathrm{GHz}$ and $40 \mathrm{GHz}$. The ratio of $\gamma_{20}$ to $\gamma_{40}$ is then used to scale the measured $20 \mathrm{GHz}$ data to $40 \mathrm{GHz}$ and compare with the measured data taken at $40 \mathrm{GHz}$. The specific attenuation $(\gamma)$ for a given drop density distribution N(D) is also a function of the wavelength $\lambda$ and the forward scattering coefficient $\operatorname{Re}\{\mathrm{S}(0)\}$ as in [6]:

$$
\gamma=4.343 \times 10^{3} \frac{\lambda^{2}}{\pi} \sum R e\{S(0)\} N(D) \Delta D
$$

where $\Delta \mathrm{D}$ is the drop size interval (in $\mathrm{mm}$ ). The forward scattering coefficient is calculated using the Mie scattering model [6], which assumes a spherical droplet shape. This computation, as well as the calculation of the drop density distribution from the binned DSD data, are detailed in [3, 7-9].

\section{B. Analysis of Rain Events}

The data used in this analysis spans from August 2014 through December 2015 and was processed to isolate applicable rain events. Events were removed from the analysis if the disdrometer was not operational, if the receivers were not operational, or if rain occurred along the path but was not measured over the site by the disdrometer (although an example of the latter is depicted for reference in Fig. 6). For each rain event, the attenuation level before and after the event was averaged and subtracted from the measured rain attenuation in an effort to isolate the excess attenuation due to rain, though this method is not perfect and other sources of attenuation (e.g. clouds) may still bias the result. Instances where this was readily apparent were removed by inspection (primarily slow-varying, high attenuation consistent with snow or water on the feed/reflector). Rainy periods were defined as any 1 minute period where the disdrometer observed more than 5 precipitating particles (roughly $0.03 \mathrm{~mm} / \mathrm{hr}$ depending on particle class), and days were included in the analysis if they included at least 10 minutes exceeding this level of activity. Altogether, 867 hours of rain events were isolated across 187 days containing rain.

\section{RESUltS}

\section{A. Time Series Results}

Time series examples are given in Figs. 4-6, comparing the measured attenuation at $20 \mathrm{GHz}$ (blue) and $40 \mathrm{GHz}$ (green) to the predicted attenuation at $40 \mathrm{GHz}$ (black) calculated by scaling the $20 \mathrm{GHz}$ data with the instantaneous scaling factor derived from the DSD. The DSD-derived attenuation is only plotted during precipitation events as described in Section III.B.

In Fig. 4, a light rain event is shown spanning approximately 8 hours. For this event, the rain rate peaked at $10 \mathrm{~mm} / \mathrm{hr}$. The attenuation predicted from the DSD-derived scaling factor tracks well with the measured attenuation for the duration of the

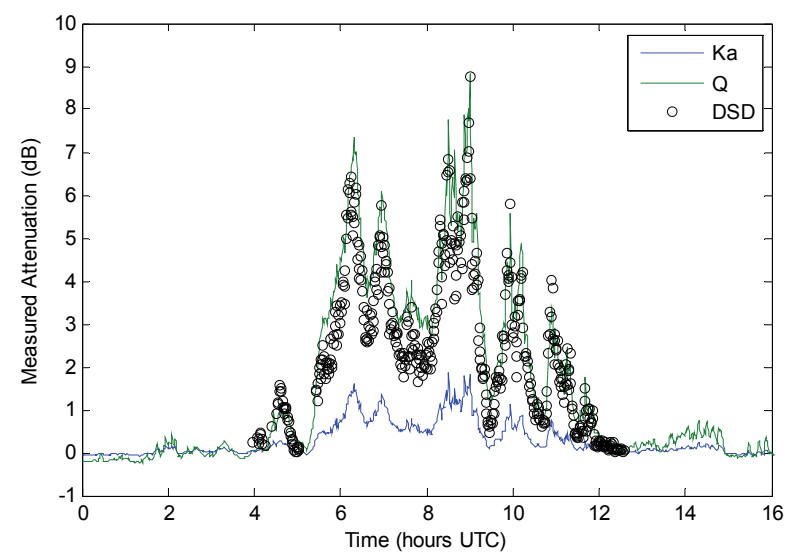

Fig. 4. Example of a light rain event (up to $10 \mathrm{~mm} / \mathrm{hr}$ ) on April $17^{\text {th }}, 2015$, comparing the measured rain attenuation to the predicted value calculated from the DSD. The prediction tracks well throughout the event.

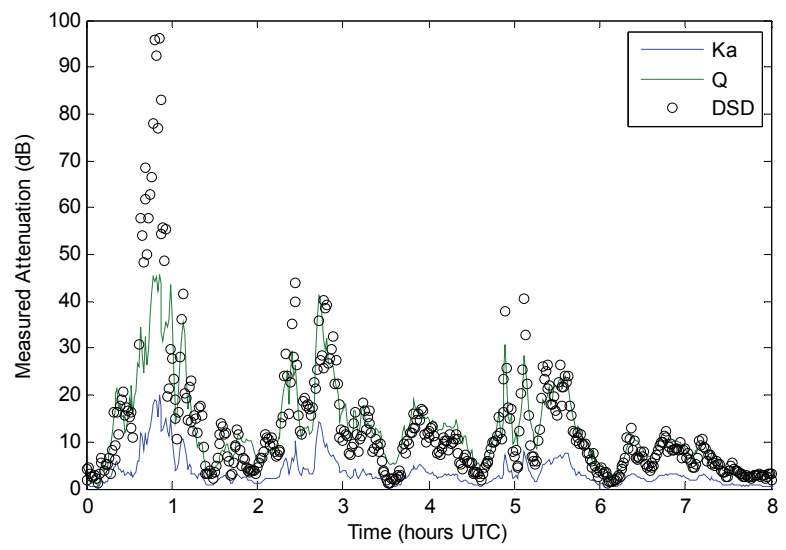

Fig. 5. Example of a heavy rain event (up to $40 \mathrm{~mm} / \mathrm{hr}$ ) on August $10^{\text {th }}$, 2015, comparing the measured rain attenuation to the predicted value calculated from the DSD. Attenuation exceeds the Q-band system dynamic range $(45 \mathrm{~dB})$ from approximately 00:45 - 01:00 UTC, while the DSD predicts attenuation up to $96 \mathrm{~dB}$ during this period.

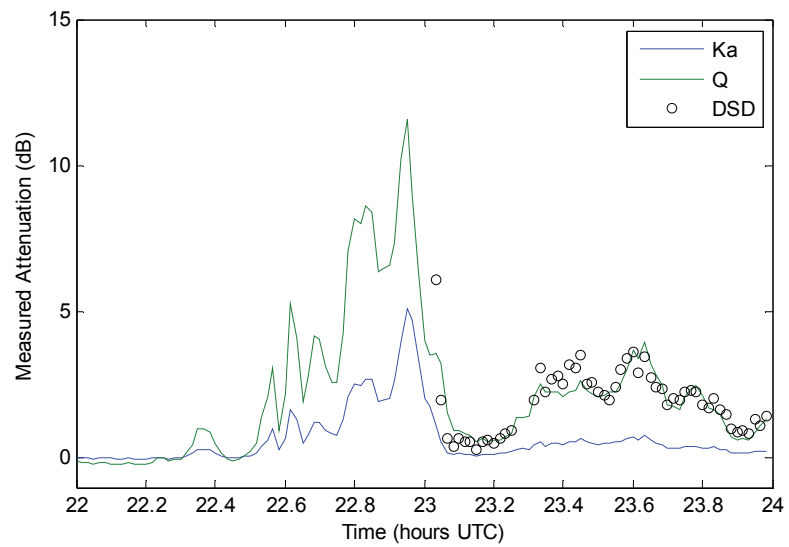

Fig. 6. Example of a light rain event (up to $10 \mathrm{~mm} / \mathrm{hr}$ ) on October $9^{\text {th }}, 2015$, comparing the measured rain attenuation to the predicted value calculated from the DSD. Attenuation up to $11.6 \mathrm{~dB}$ is measured during the first half of the event, but no rain is measured at the site, indicating that the rain is along the path. By the end of the event, the rain cell appears to have moved overhead the receiver, and the DSD prediction tracks the measurement. 

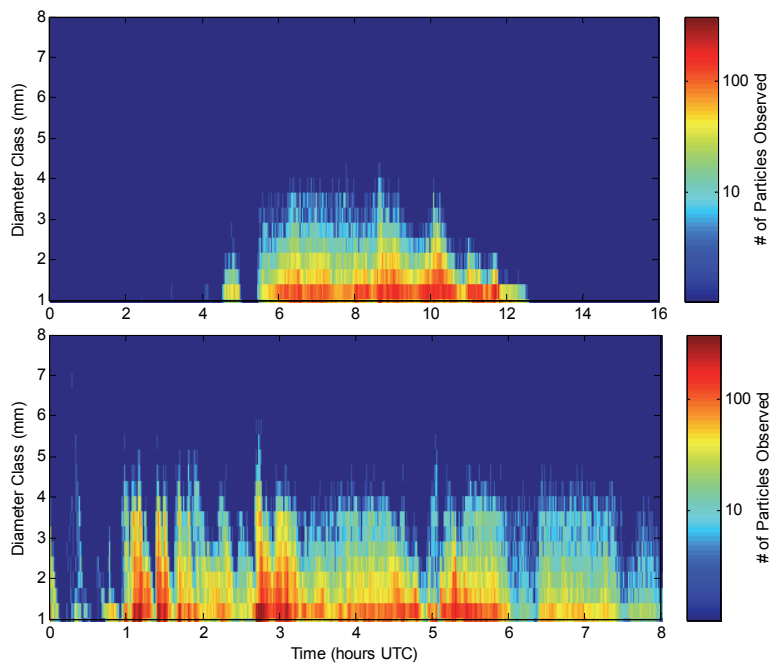

Fig. 7. The drop size distribution as a function of time. In the above plot, the DSD throughout the light rain event of Fig. 4. Below, the DSD during the heavy rain event of Fig. 5 .
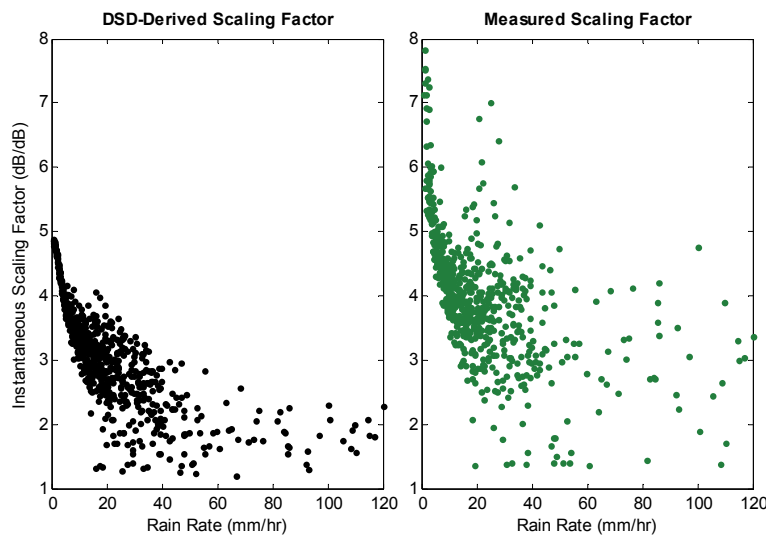

Fig. 8. The average instantaneous scaling factor for a given rain rate, as derived from the DSD (left) and as measured by the receivers (right). As shown, the scaling factor is averaged across rain rate bins of $0.05 \mathrm{~mm} / \mathrm{hr}$.

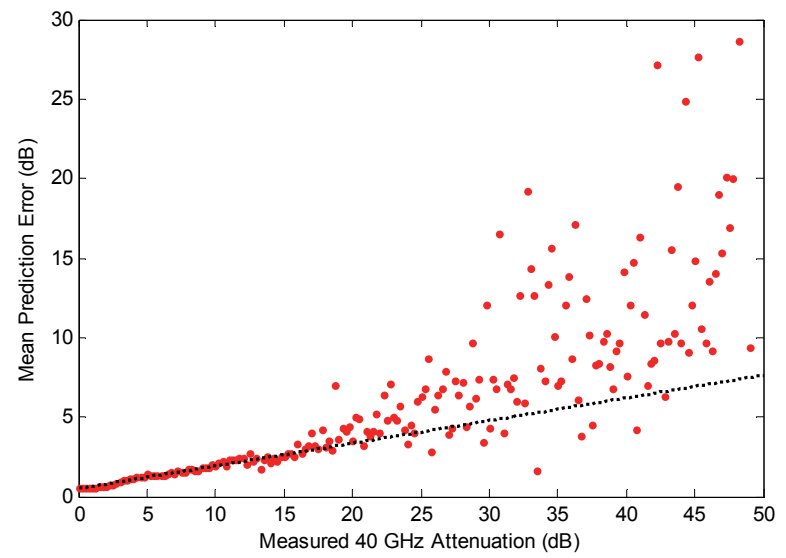

Fig. 9. The error in the DSD-predicted attenuation as a function of the measured attenuation level. A linear fit from $0-15 \mathrm{~dB}$ indicates a trend of $0.14 \mathrm{~dB}$ error per $\mathrm{dB}$ of attenuation. Variance of the prediction error increases as attenuation approaches the system dynamic range of $40 \mathrm{~dB}$. event, with a mean error of $0.58 \mathrm{~dB}$ and a maximum error of $2.42 \mathrm{~dB}$.

In Fig. 5, a heavy rain event is shown, also spanning approximately 8 hours. Here, the rain rate reaches up to 40 $\mathrm{mm} / \mathrm{hr}$. The predicted attenuation generally tracks well with the measured attenuation, but for approximately 15 minutes shortly before 1:00 UTC, the total attenuation exceeds the dynamic range of the Q-band system $(40 \mathrm{~dB})$, saturating the measurement around $45 \mathrm{~dB}$ while the scaled attenuation peaks at $96 \mathrm{~dB}$. Throughout the even, a mean error of $3.64 \mathrm{~dB}$ is observed with a maximum error of $51.33 \mathrm{~dB}$ during the period where the dynamic range is exceeded.

In Fig. 6, another light rain event is shown, but in this case the event begins along the path and precipitation is not immediately measured by the disdrometer. About half an hour into the event, the rain cell has moved over the receiver and precipitation is measured, resulting in the plotted DSD-derived prediction. As detailed in Section III.B, the first half of this event would be excluded from the statistical analysis, but the second half is included where disdrometer measurements are available. During the measured portion of the event, the mean prediction error was $0.35 \mathrm{~dB}$ with a maximum error of $2.54 \mathrm{~dB}$.

\section{B. Temporal Variability of Drop Size Distribution}

To demonstrate the temporal variability of the drop size distribution and its relation to attenuation, Fig. 7 plots the DSD over time during the light rain event of Fig. 4 (above) and the heavy rain event of Fig. 5 (below). Generally speaking, the more intense periods of DSD correlate to a higher rain rate and higher attenuation. The DSD is visibly more variable throughout the heavy rain event as compared to the light rain event.

\section{Instantaneous Scaling Factor vs. Rain Rate}

The instantaneous scaling factor is plotted against rain rate in Fig. 8, as derived from both the DSD (left) and the measured attenuation levels (right). As shown, all scaling factors observed in the 17 month observation period are averaged across rain rate bins of $0.05 \mathrm{~mm} / \mathrm{hr}$. Both the measured and DSD-derived instantaneous scaling factor decrease as rain rate increase, and the variance of the scaling factor also increases with increasing rain rate. For rain rates below $5 \mathrm{~mm} / \mathrm{hr}$, the mean instantaneous scaling factor is $4.31 \mathrm{~dB} / \mathrm{dB}$ as derived from the $\mathrm{DSD}$, and 5.88 $\mathrm{dB} / \mathrm{dB}$ as observed from the measured attenuations.

\section{Prediction Error vs. Attenuation}

The error of the DSD-derived scaled attenuation as compared to the measured attenuation increases linearly as a function of attenuation level, as demonstrated in Fig. 9. All prediction error observations over the 17 month period are included and averaged across bins of $0.25 \mathrm{~dB}$ attenuation level. A linear fit from $0-15 \mathrm{~dB}$ indicates a trend of $0.14 \mathrm{~dB}$ prediction error for every $1 \mathrm{~dB}$ of rain attenuation. Beyond 15 $\mathrm{dB}$, the noise of the prediction error increases rapidly; the ability to characterize the true prediction error deteriorates beyond this point, as the calculated error is prone to increase drastically as the measured attenuation approaches the system dynamic range of $40 \mathrm{~dB}$ and the measurement not only saturates, but becomes noisier due to influence of the system noise floor. 


\section{E. Attenuation vs. Mean Drop Diameter and Velocity}

Lastly, Fig. 10 and Fig. 11 plot the measured Q-band attenuation against the mean droplet diameter and mean droplet velocity, respectively, for all observations from August 2014 through December 2015. As the scatter plots show, higher attenuation values almost exclusively occur toward the center of the bell curve of particle diameter and velocity. Across all observations, the total mean particle diameter was $0.40 \mathrm{~mm}$, and total mean velocity was $1.91 \mathrm{~m} / \mathrm{s}$. Above $15 \mathrm{~dB}$ of attenuation, the particle diameter generally remained within $0.15 \mathrm{~mm}$ of the mean diameter, and within $0.5 \mathrm{~m} / \mathrm{s}$ of the mean velocity.

\section{CONCLUSIONS}

As communications systems advance to higher frequencies, attenuation predictions are necessary and may often be derived from scaling of existing data at lower frequencies. Herein, we investigated the use of the drop size distribution from an optical disdrometer to derive an instantaneous scaling factor, and thereby predict rain attenuation at $40 \mathrm{GHz}$ by scaling measured rain attenuation at $20 \mathrm{GHz}$. Time series examples of the scaled result were presented for different types of rain event, and various aspects of the approach were explored: the temporal variability of the drop size distribution within different types of events, the trend of the instantaneous scaling factor as a function of the rain rate, and the error of the scaled attenuation prediction as a function of total attenuation level. The correlations between measured attenuation and the mean drop size and mean drop velocity were also investigated. This analysis indicates that the instantaneous scaling factor decreases as a function of rain rate, while its variance increases as a function of rain rate. Similarly, the error of the scaled predicted attenuation increases linearly as a function of attenuation level -- a linear trend of $0.14 \mathrm{~dB}$ error per $\mathrm{dB}$ attenuation. It was also observed that higher attenuations mostly occur with droplet diameter and velocity near the mean.

In terms of future work, NASA GRC has also deployed a laser disdrometer in conjunction with an ongoing $\mathrm{V} / \mathrm{W}$-band terrestrial link experiment at 72 and $84 \mathrm{GHz}$ in Albuquerque, NM. This affords the opportunity to conduct a similar analysis of the instantaneous scaling factor at these frequencies, and investigate the relation to the scaling factor at $\mathrm{Ka}$ and Q-band.

\section{REFERENCES}

[1] D. de Wolf, "On the Laws-Parsons distribution of raindrop sizes," Radio Science, Vol. 36, No. 4, pp.639-642, July 2001 .

[2] J. Nessel, M. Zemba, L. Luini, C. Riva, "Comparison of Instantaneous Frequency Scaling from Rain Attenuation and Optical Disdrometer Measurements at K/Q bands", $21^{\text {st }}$ $\mathrm{Ka}$ and Broadband Communications Conference, Bologna, Italy, October 2015.

[3] M. Zemba, J. Morse, J. Nessel, "Frequency Estimator Performance for a Software-based Beacon Receiver," IEEE Antennas and Propagation Symposium, July 2014, pp.1574-1575.

[4] J. Nessel, J. Morse, M. Zemba, C. Riva, L. Luini, "Preliminary Results of the NASA Beacon Receiver for the Alphasat Aldo Paraboni TDP5 Propagation Experiment," $20^{\text {th }} \mathrm{Ka}$ and Broadband Communications Conference, Salerno, Italy, October 2014.

[5] Thies Clima Laser Precipitation Monitor: Instructions for Use. Rev. 2.5. July 2011.

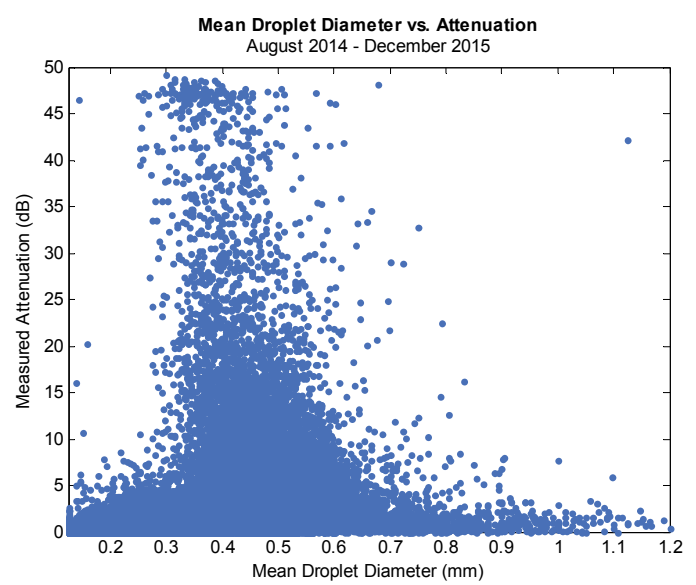

Fig. 10. Scatter plot of the measured Q-band attenuation and the mean droplet diameter for all observations from August 2014 - December 2015.

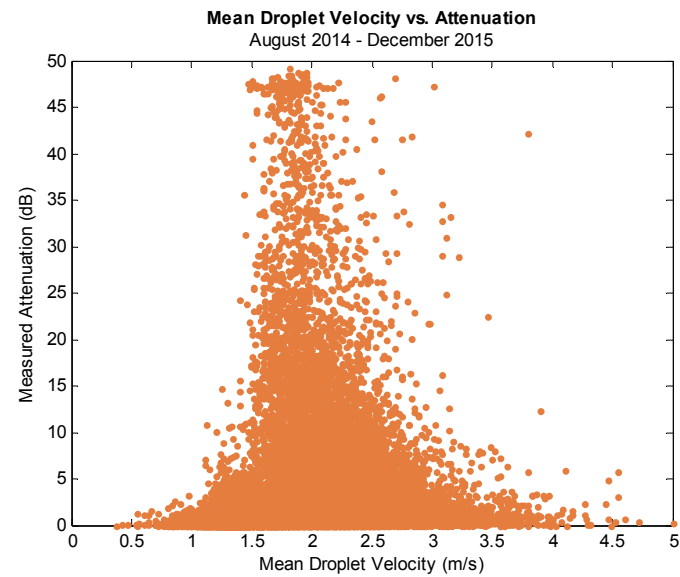

Fig. 11. Scatter plot of the measured Q-band attenuation and the mean droplet velocity for all observations from August 2014 - December 2015.

[6] M. Sadiku, Numerical Techniques in Electromagnetics, $2^{\text {nd }}$ Ed., CRC Press, 2001.

[7] C. Bohren, D. Huffman, Absorption and Scattering of Light by Small Particles, Wiley, 1983.

[8] H.Y. Lam, J. Din, L. Luini, A. Panagopoulos, C. Capsoni, "Analysis of Raindrop Size Distribution Characteristics in Malaysia for Rain Attenuation Prediction," 2011 URSI General Assembly and Scientific Symposium, August 2011.

[9] R. Gunn, G.D. Kinzer, "The terminal velocity of fall for water droplets in stagnant air," Journal of Meteorology, Vol. 8, pp.249-253, 1949.

[10] S. Bertorelli and A. Paraboni, "Modelling of short-term frequency scaling for rain attenuation using ITALSAT data," Int. J. Satell. Commun., vol. 25, no. 3, pp. 251-262, 2007.

[11] G. Karagiannis, A. D. Panagopoulos, J. D. Kanellopoulos, "Short-Term Rain Attenuation Frequency Scaling for Satellite Up-Link Power Control Applications", IEEE Transactions on Antennas and Propagation, vol.61, Issue 5, May 2013.

[12] D.E. Setzer, "Computer transmission through rain at microwave and visible frequencies," Bell Syst. Tech. J, vol. 49 , no. 8 , Oct. 1970 , pp.1873-1892. 